\title{
An Approach to Ranking Integrated Models for Risk Assessment by Using the Internet of Intelligences
}

\author{
Weidan Wang ${ }^{1,2}$, Sen Qiao ${ }^{3}$, Fanlei Zeng ${ }^{1,2}$, Jun Guo ${ }^{1,2}$, Chongfu Huang, ${ }^{1,2, *}$ \\ ${ }^{1}$ Key Laboratory of Environmental Change and Natural Disaster, Ministry of Education of China, \\ Beijing Normal University, Beijing 100875, China \\ ${ }^{2}$ Academy of Disaster Reduction and Emergency Management, Ministry of Civil Affairs \& Ministry of \\ Education, the Peoples' Republic of China, Beijing 100875, China \\ ${ }^{3}$ Institute of Geophysics, China Earthquake Administration, Beijing 100081, China
}

Received 19 February 2016

Accepted 2 March 2016

\begin{abstract}
In natural disaster risk assessment, it is referred to as integrated model which is used to integrate information. The statistical model is essentially integrated model. Usually, people try to search the reasons that choose a model from the theory or case. Based on the thinking of experimental riskology, this paper puts forward with the idea that an experimental platform, which is used to test integrated models, can be built with the Internet of intelligences. In this way, models can be ranked, that provides some reference for the users. In this paper, the models of analytic hierarchy process, the median method corresponding to time and comprehensive evaluation method are used to integrate the useful information provided by three agents online in the gobang game, and then play with the computer. Through analyzing the results of Human-Computer Fighting people can judge which model is better. The experimental results show that for gobang, the effect of simple comprehensive evaluation method is better than analytic hierarchy process.
\end{abstract}

Keywords: Risk assessment, information integration, experimental riskology, internet of intelligences, gobang

\section{用智联网对整合型风险评估模型进行排序的一条途径}

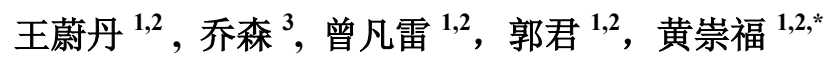

1. 北京师范大学环境演变与自然灾害教育部重点实验室，北京 100875 , 中国 2.民政部/教育部减灾与应急管理研究院，北京 100875 , 中国

3.中国地震局地球物理研究所, 北京 100081 , 中国

摘要：在自然灾害风险评估中, 用于整合信息的模型, 称为整合模型。统计模型, 本质上是整合模 型。通常, 人们从理论或案例中寻找为什么使用某个模型的理由。基于实验风险学的思考, 本文提出用 智联网搭建实验平台, 对整合型模型进行测试, 从而对参与实验的模型进行优劣排序, 供使用者参考。 本文分别将层次分析法、时间中值法和综合评判法用于整合五子棋游戏中三位选手的落子，与计算机博 弯, 用战绩来测试这三种模型的效果。实验表明, 对五子棋而言, 简单的综合评价法比层次分析法效果 好。

关键词: 风险评估, 信息整合, 实验风险学, 智联网, 五子棋

\section{1. 引言}

自然灾害风险评估, 是对自然事件或力量为 主因导致的未来不利事件情景进行的评定和估
计。用数理统计方法处理历史灾害资料, 估算某 种期望值作为风险值, 是最常见的资料整合法。 既不考虑灾害发生的机理, 也不考虑风险的动态 性, 用综合评价法或层次分析法综合专家意见, 是最常见的经验整合法。

\footnotetext{
* Corresponding Author: hchongfu@bnu.edu.cn
} 
更一般地讲, 完整刻画自然灾害风险, 必须 涉及风险的空间位置、水平高低和有效时段。如 果能建立自然灾害系统的微分方程, 例如某类广 义力学系统的能量方程 ${ }^{[1]}$, 并考虑风险系统的种 种不确定性 ${ }^{[2,3]}$, 完整刻画风险并不是问题。无论 是从能量平衡方程导出的气候模式, 还是弹塑性 力学的地震波动方程, 抑或是欧洲开发的关于酸 雨污染综合分析的计算机模型 RAINS, 其实都是 在往这个方向努力。尽管人们已经探讨用微分方 程帮助研究口蹄疫动态影响的风险问题 ${ }^{[4]}$, 用药 代动力学模型研究贞幼儿使用灭活流感疫苗的 风险问题 ${ }^{[5]}$, 但诸如癌症风险这类问题, 泛泛地 讨论所谓的微分方程, 都非常困难, 概率风险评 估仍是唯一有效的方法 ${ }^{[6]}$ 。今天, 以代数方程求 解 ${ }^{[7]}$ 、有限差分 ${ }^{[8,9]}$ 、小波变换 ${ }^{[10]}$ 等方法, 并借助 云计算工具 ${ }^{[1]]}$, 求解超大型微分方程已不是问 题。然而, 即使自然定律对我们已无秘密可言, 我们也只能近似地知道初始状态和边界条件。况 且, 风险问题涉及人类社会的财产和生命, 随机 微分方程式的自然灾害动态风险分析, 目前只能 是一个图腾。

现今的自然灾害风险评估方法, 大多涉及用 已观测的样本来计算致灾因子的概率密度分布, 其前提是假定了相应的风险系统之变化是平稳 马尔可夫随机过程。但事实是: 风险系统不仅涉 及到自然因素, 社会系统更是在其中占据重要位 置; 而多数社会系统并不能用平稳马尔科夫过程 描述。人们花费巨大人力、物力、财力制作出来 的所谓 “自然灾害风险图”, 却不能从理论上证 明其结果是可信的。为此, 人们提出了 “实验风 险学” 概念, 力图通过实验来检验相关的风险评 估方法, 研究风险的本原, 解决风险分析可靠性 问题 ${ }^{[12]}$ 。伴随着互联网的飞速发展, 建立网上实 验平台, 研究各种模型的可靠性, 成为可能。“智 联网” 的提出, 为建立计算机网络实验平台, 提 供了重要的工具 ${ }^{[13]}$ 。

最著名的计算机实验平台, 莫过于美国 IBM 公司的计算机 “深蓝” 和 “沃森”, 它们通过与 国际象棋世界冠军的对弯和击败智力游戏节目 中人类冠军, 分别展现了计算机的强大能力和许 多诸如 “嵌套分解” 算法的优越性。

本文首次提出并实践了在智联网平台上对 整合型模型进行测试, 对模型进行优劣排序, 为 检验风险评估模型提供了一种途径。

\section{2. 实验风险学的基本思想}

风险学不是一门纯理论的科学, 而是一门应 用科学。风险学成熟的标志不仅是数学化, 而且 是实验化, 并且要求实验的结果能被别人重复。 今日之经济学之所以不成熟, 并不是数学化不 够, 而是缺乏实验手段的支持, 所以经济学家们 对经济危机是否出现、程度如何, 总是莫衷一是。 当人们不满足在自然条件下去观察对象, 要求对 被研究对象进行积极的干预时, 就导致科学实验 的产生。

以往, 人们通过实验所要认识的对象是自然 界的物体 (例如: 机床、建筑物) 或现象 (例如: 太阳光、地振动)。模型实验产生以后, 人们用 模型来代替原型进行实验。今天, 我们通过实验 所要认识的对象是 “风险”, 它是与某种不利事 件有关的一种未来情景。这种对象既不是自然界 的物体, 也不是自然界的现象, 而是与人类社会 息息相关, 并受人类社会变化而影响的一种特殊 现象。我们不可能建立一种实验理论和方法对任 意的风险现象进行实验研究。不同的风险现象会 有不同的实验模型。

传统的物理实验, 可用于伪风险实验。这类 风险可以用系统模型和现有数据精确预测。例 如, 爆炸实验, 足可验证爆炸模型是否精确; 传 统的随机实验, 可用于概率风险实验。可以用概 率模型和大量数据进行统计预测的与特定不利 事件有关的未来情景称为概率风险。例如, 建筑 物在地震波作用下的破坏实验, 足可验证建筑物 概率下的震害预测模型是否可靠。对于只能用模 糊逻辑和不完备信息近似推断出的模糊风险, 目 前尚无可用的实验方法。而用现有方法不可能预 测和推断的不确定风险, 相关实验, 或许只能是 某种类比, 寻找一些未来情景的蛛丝马迹。

面对瞬息万变又充满危险的世界, 人们不可 能极端精确地、过分仔细地寻找风险系统状态方 程, 更不可能等待很长时间获取足够的统计资料 后较精确地估计概率风险。使用有限的知识和资 料快速进行风险评估, 得出的必然是模糊风险。

人们评估出的模糊风险, 依据的是不完备信 息, 大多涉及主观判断。通过实验, 有助于检验 其所用模型是否合理。这样的实验, 少不了人员 的参与。原则上讲, 有人员参与的实验, 完全可 以通过手工完成。智联网的出现, 则可大大降低 这类实验的成本, 提高实验的效率。 


\section{3. 智联网基本概念}

智联网是一个生产智慧产品的网络, 由多个 智能体, 经互联网连接, 使用某种数学模型激发 智能体的知识和经验, 并进行智力提升式整合。 最简单的智联网可以用图 1 示之, 该系统由三个 智能体 $a_{1}, a_{2}, a_{3}$, 三台终端机 $c_{1}, c_{2}, c_{3}$, 一台服务 器 $S$ 和一个信息处理模型 $M$ 组成。

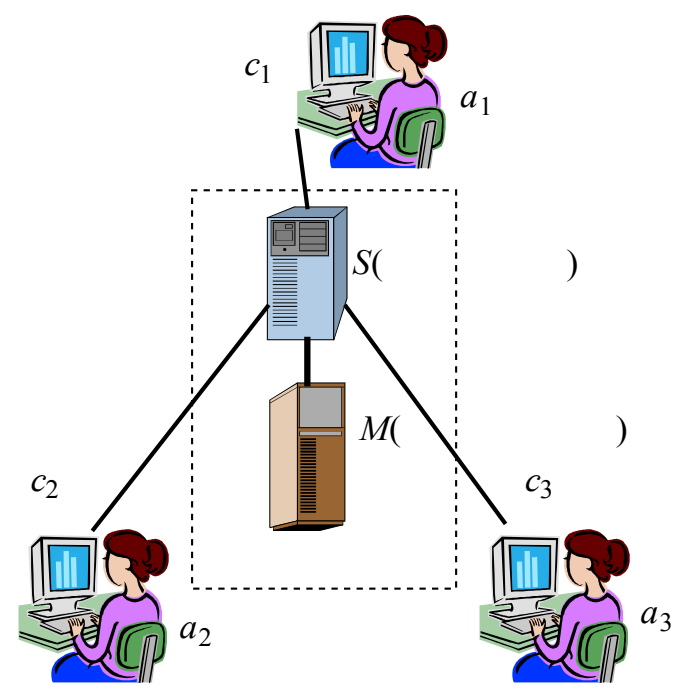

图 1. 最简单的智联网。图中的三台计算机形成 网络, 三个工作人员是三个智能体。

事实上, 并非智能体联网就能形成智联网。 例如, 医学影像远程网络会诊并不是智联网, 因 为系统中并不存在本质上能对专家意见进行处 理的任何模型。并非智联网均有正向性, 例如, 用一个简单的统计模型支撑的智联网, 理论上来 讲, 智力水平为网中智能体的平均值。显然, 智 联网的核心技术在于网络信息处理模型 $M$ 。智联 网形式化定义如下 ${ }^{[13]}$ :

定义 1 设 $A$ 是一个智能体集合, $N$ 是 $A$ 使 用的一个网络, $M$ 是处理 $A$ 所提供信息的模型, 三元体 $<A, N, M>$ 称为一个智联网, 记为 $\Phi$ 。

智联网的工作原理是, 客户发布问题, 智能 体根据自己掌握的信息和经验, 在线解答问题。 智联网用模型来自动处理并整合多个智能体的 解答, 形成优化答案。例如, 北京某考生的高考 模拟成绩为 600 分, 其报清华、北大的风险有多 大? 过来人的经历和长者的经验由智联网数据 化后再处理而生成的正反风险分析报告就极具 价值。如果报而落选, 此为正风险; 如果转而填 报其他学校而明显损失高考分数, 此为负风险。

客户发布问题和智能体的响应自动数据化,
是智联网中的数学模型能发挥作用的关键, 也是 其强于现有网页语意分析技术的独到之处。恶意 虑假信息过滤技术和不协调信息感知技术是智 联网的必备技术，而信息扩散技术则是智联网的 核心技术。

智联网与网络调查最大的不同是, 智联网并 不限于用统计意义的结果服务客户, 更多的是情 景比对和过来人的量化经验。智联网系统更不是 网上自动办公系统, 而是一个超级智慧生产系 统, 必须有智能化的数学处理模型 $M$ 。

显然, 如果能通过实验对答案的优化度进行 测试, 就能判断整合模型的优劣。

本文用智联网平台上五子棋的对亦实验, 由 战绩来测试整合模型的优劣。

\section{4. 实验设计及过程}

根据智联网的工作原理, 首先明确要解决的 风险问题, 然后各个智能体根据自己掌握的经验 和信息, 在智联网实验平台上解答问题。最后, 智联网选用合适的模型来处理、整合多个智能体 的解答, 给出最终方案。本实验采用三人智联网 与计算机进行五子棋对弯的方式进行。用对弯输 赢来评判智联网所用模型的优劣。被评判的是层 次分析法、时间中值法和综合评判法。

之所以选择五子棋, 是因为它简单灵活, 容 易上手, 一方五子连成一线就赢了。实验系统是 一个 Win32 系统下以 $\mathrm{C}$ 语言开发的桌面应用程 序。电脑方使白子, 智联网方使黑子且先落子。

\section{1 设计思路}

三个以智能体身份参加实验的在读博士研 究生分别记为 $a_{1}, a_{2}, a_{3}$ 。智联网中的模型整合 $a_{1}, a_{2}, a_{3}$ 的落子建议, 生成落子执行指令, 与 计算机对弯。用各模型分别与计算机对亦 5 局。 实验遵循以下 3 个原则:

1)一局结束, 如果黑方 (智能体方) 胜, 则 闯关成功。

2)闯关成功越多的模型效果越好。

3)考虑智能体的水平因素, 可能造成采用不 同模型时输赢次数相当的情况, 将下棋步数、时 间考虑在内，作为评判的另外两个指标。

\section{2 三整合模型简介}

\subsection{1 层次分析法}

层次分析法是一种定性和定量相结合的、系 
统化、层次化的分析方法, 利用层次分析法把智 能体 $a_{1}, a_{2}, a_{3}$ 的决策结果进行综合, 综合后的 结果即为黑子落棋位置。

假设 3 个智能体所建议的落子坐标为 $(9,9)$ $(9,8)(9,7)$, 用层次分析法整合, 生成落子执 行指令的坐标将是 $(8,8)$ 。我们通过下面的算法
素对目标层 $Z$ 的影响两两比较结果如表 1 所示, 其中 $Z_{12}=9 / 8$, 表示 $C_{1}$ 对目标层 $Z$ 闯关成功的重 要程度略高于 $C_{2}$, 即进攻相对于防守要更为重要 些。

对应矩阵的最大特征根为 $\lambda=2$, 归一化特征 向量为:

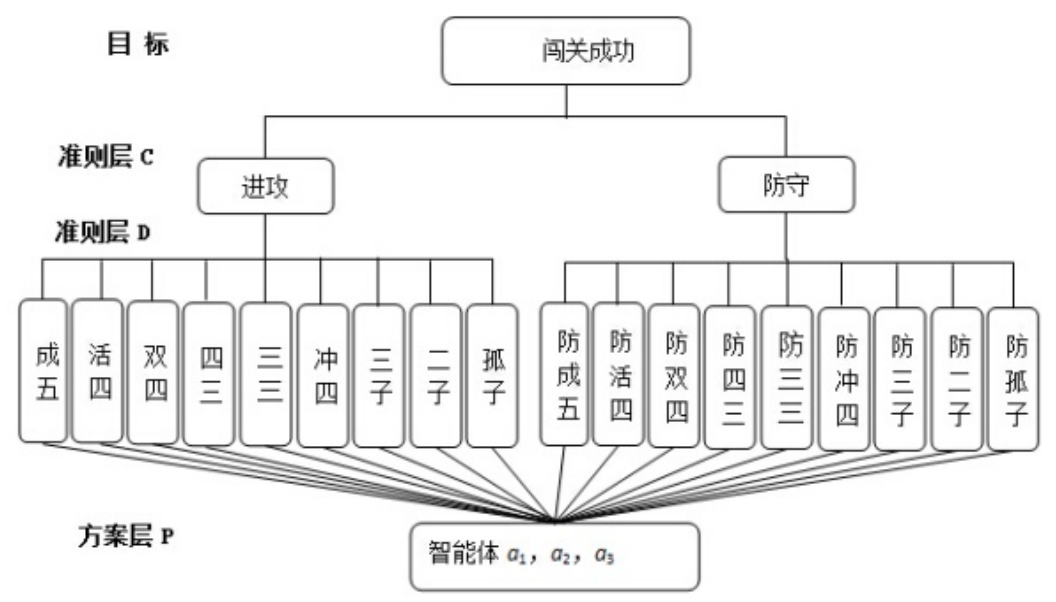

图 2. 五子棋 AHP 层次结构模型.

来实现这种整合。

建立层次结构模型。五子棋 AHP 层次结构 模型如图 2 所示, 根据五子棋闯关这个具体问题 把相关的各个因素分为目标层 $Z$ 、准则层 $C$ 、准 则层 $D$ 、方案层 $P$ 。其中目标为闯关成功, 即智 能体和计算机下五子棋, 赢过计算机。闯关成功 的影响因素为进攻和防守, 这就是准则层 $C$, 进 攻又分为成 “成五”、“活四”、“双四”、“四 三”、“三三”、“冲四”、“三子”、“二子”、 “孤子” 等等, 组成准则层 $C$ 的子因素。“成 五” 指的是落子后己方要五子连珠, 赢了。“活 四” 指的是有两个点可以成五的四。“双四” 是 指一子落下同时形成两个 “冲四”。“四三” 指 一子落下同时形成一个 “冲四” 和一个 “活 三”。“三三” 是一子落下同时形成两个 “活 三”。其中 “冲四” 指只有一个点可以成五的 四, “活三” 则是再走一着可以形成 “活四” 的 三。“眠三” 和 “活三” 统称为 “三子” , “眠 三” 指再走一着可以形成 “冲四” 的三。“二 子” 指的是在一条阳线或阴线上连续相邻的 5 个 点上只有两枚同色棋子的棋型。棋盘上的横纵直 线称阳线, 阳线垂直相交的点相互之间连接形成 与阳线 $45^{\circ}$ 的隐形斜线, 称为阴线。孤子则指该 子和前面所下子没有关系, 或者是开局。落子在 $(9,8)$ 指的是落在第 9 列, 第 8 行, 即图 3 中 黑色圆圈所表示的位置。

构造判断矩阵及层次单排序。准则层 $C$ 的要

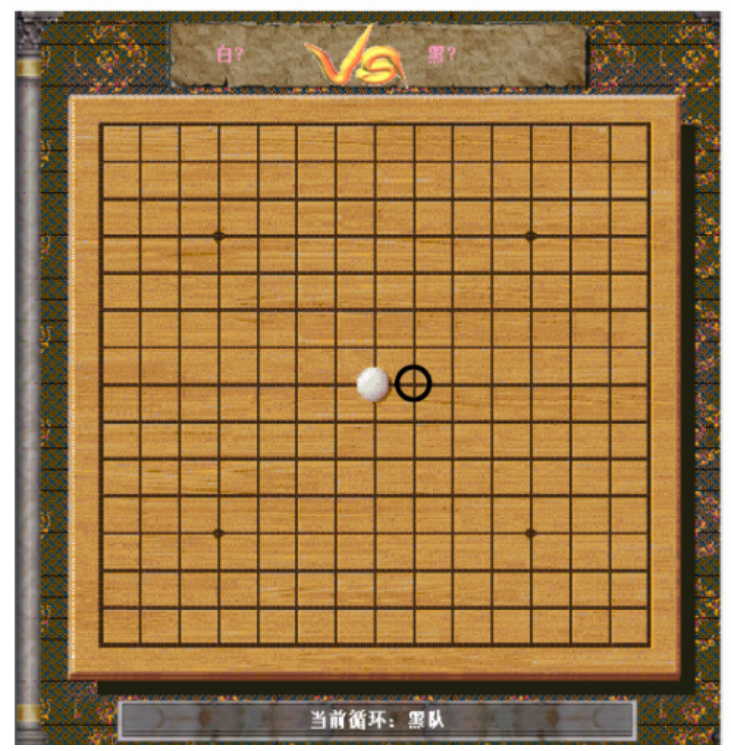

图 3. 五子棋棋盘.

$$
\omega=\left(a_{1}, a_{2}\right)=\left[\begin{array}{l}
0.5294 \\
0.4706
\end{array}\right]
$$

式中的 $\omega$ 表示同一层次因素对于上一层次某因 素相对重要性的排序权值, 这一过程称为层次单 排序。 
表 1. 准则层 $C$ 对目标层 $Z$ 的影响

\begin{tabular}{lll}
\hline$Z$ & $C_{1}$ & $C_{2}$ \\
\hline$C_{1}$ & 1 & $9 / 8$ \\
$C_{2}$ & $8 / 9$ & 1 \\
\hline
\end{tabular}

用 $C I=\frac{\lambda-\mathrm{n}}{n-1}$ 对 $\lambda$ 进行一致性检验, 其中 $n$ 是表 1 中对角线元素之和。 $C I=0$, 表示通过 了一致性检验。

同理, 准则层 $D$ 中对应 $C_{1}$ 的各要素对准则 层要素 $C_{1}$ 的影响两两比较可得表 2 。

表 2. 准则层 $D$ 的各要素对准则层要素 $C_{1}$ 判断矩阵

\begin{tabular}{cccccccccc}
\hline$C_{1}$ & $D_{1}$ & $D_{2}$ & $D_{3}$ & $D_{4}$ & $D_{5}$ & $D_{6}$ & $D_{7}$ & $D_{8}$ & $D_{9}$ \\
\hline$D_{1}$ & 1 & 3 & 3 & 4 & 5 & 6 & 7 & 8 & 9 \\
$D_{2}$ & $1 / 3$ & 1 & 1 & $4 / 3$ & $5 / 3$ & 2 & $7 / 3$ & $8 / 3$ & 3 \\
$D_{3}$ & $1 / 3$ & 1 & 1 & $4 / 3$ & $5 / 3$ & 2 & $7 / 3$ & $8 / 3$ & 3 \\
$D_{4}$ & $1 / 4$ & $3 / 4$ & $3 / 4$ & 1 & $5 / 4$ & $3 / 2$ & $7 / 4$ & 2 & $9 / 4$ \\
$D_{5}$ & $1 / 5$ & $3 / 5$ & $3 / 5$ & $4 / 5$ & 1 & $6 / 5$ & $7 / 5$ & $8 / 5$ & $9 / 5$ \\
$D_{6}$ & $1 / 6$ & $3 / 6$ & $1 / 2$ & $4 / 6$ & $5 / 6$ & 1 & $7 / 6$ & $4 / 3$ & $3 / 2$ \\
$D_{7}$ & $1 / 7$ & $3 / 7$ & $3 / 7$ & $4 / 7$ & $5 / 7$ & $6 / 7$ & 1 & $8 / 7$ & $9 / 7$ \\
$D_{8}$ & $1 / 8$ & $3 / 8$ & $3 / 8$ & $4 / 8$ & $5 / 8$ & $6 / 8$ & $7 / 8$ & 1 & $9 / 8$ \\
$D_{9}$ & $1 / 9$ & $1 / 3$ & $1 / 3$ & $4 / 9$ & $5 / 9$ & $6 / 9$ & $7 / 9$ & $8 / 9$ & 1 \\
\hline
\end{tabular}

对应矩阵的最大特征根为 $\lambda=9$, 对应的特征 特征向量归一化结果为

$$
\vartheta_{1}=\left(b_{1}, b_{2}, \cdots, b_{9}\right)^{T}=\left[\begin{array}{l}
0.3756 \\
0.1252 \\
0.1252 \\
0.0939 \\
0.0751 \\
0.0626 \\
0.0537 \\
0.4670 \\
0.0417
\end{array}\right]
$$

同样可对其进行一致性检验, $C I=\frac{\lambda-\mathrm{n}}{n-1}, n$ 为 表 2 对角线元素之和。 $C I=0$, 表示通过了一致 性检验。准则层 $D$ 中对应 $C_{2}$ 的各要素对准则层 要素 $C_{2}$ 的影响两两比较可得表 3 。

特别值得注意的是, 因为进攻 $\left(C_{1}\right)$ 和防守 $\left(C_{2}\right)$ 是相对的, 准则层 $D$ 中的“成五” $\left(D_{1}\right)$ 和 “防成五” $\left(D_{10}\right)$ 是相对的, “活四” $\left(D_{2}\right)$ 和“防 活四” $\left(D_{11}\right)$ 是相对的, 那么“成五”、“活四”对
于进攻的相对重要性和“防成五”、“防活四”对于 防守的相对重要性是同样的, 其它要素同理, 进 攻和防守相对应的下级要素是对称的, 所以表 2 和表 3 中的数值相同。

表 3. 准则层 $D$ 的各要素对准则层要素 $C_{2}$ 判断矩阵

\begin{tabular}{cccccccccc}
\hline$C_{2}$ & $D_{10}$ & $D_{11}$ & $D_{12}$ & $D_{13}$ & $D_{14}$ & $D_{15}$ & $D_{16}$ & $D_{17}$ & $D_{18}$ \\
\hline$D_{10}$ & 1 & 3 & 3 & 4 & 5 & 6 & 7 & 8 & 9 \\
$D_{11}$ & $1 / 3$ & 1 & 1 & $4 / 3$ & $5 / 3$ & 2 & $7 / 3$ & $8 / 3$ & 3 \\
$D_{12}$ & $1 / 3$ & 1 & 1 & $4 / 3$ & $5 / 3$ & 2 & $7 / 3$ & $8 / 3$ & 3 \\
$D_{13}$ & $1 / 4$ & $3 / 4$ & $3 / 4$ & 1 & $5 / 4$ & $3 / 2$ & $7 / 4$ & 2 & $9 / 4$ \\
$D_{14}$ & $1 / 5$ & $3 / 5$ & $3 / 5$ & $4 / 5$ & 1 & $6 / 5$ & $7 / 5$ & $8 / 5$ & $9 / 5$ \\
$D_{15}$ & $1 / 6$ & $3 / 6$ & $1 / 2$ & $4 / 6$ & $5 / 6$ & 1 & $7 / 6$ & $4 / 3$ & $3 / 2$ \\
$D_{16}$ & $1 / 7$ & $3 / 7$ & $3 / 7$ & $4 / 7$ & $5 / 7$ & $6 / 7$ & 1 & $8 / 7$ & $9 / 7$ \\
$D_{17}$ & $1 / 8$ & $3 / 8$ & $3 / 8$ & $4 / 8$ & $5 / 8$ & $6 / 8$ & $7 / 8$ & 1 & $9 / 8$ \\
$D_{18}$ & $1 / 9$ & $1 / 3$ & $1 / 3$ & $4 / 9$ & $5 / 9$ & $6 / 9$ & $7 / 9$ & $8 / 9$ & 1 \\
\hline
\end{tabular}

对应矩阵的最大特征根为 $\lambda=9$, 归一化特征 特征向量为

$$
\vartheta_{2}=\left(b_{10}, b_{11}, \cdots, b_{18}\right)^{T}=\left[\begin{array}{c}
0.3756 \\
0.1252 \\
0.1252 \\
0.0939 \\
0.0751 \\
0.0626 \\
0.0537 \\
0.4670 \\
0.0417
\end{array}\right]
$$

同样可通过 $C I=0$ 的一致性检验。根据智能 体 $a_{1}, a_{2}, a_{3}$ 落子坐标 $(9,9)(9,8)(9,7)$ 及现 有的格局, 调用程序对每个落子对准则层 D 中五 子、活四、双四等的贡献进行判断, 生成各自的 判断矩阵。设对应生成的判断矩阵为:

$$
\rho=\left(c_{1 j}, c_{2 j}, c_{3 j}\right)^{T}, j=1,2, \cdots, 18
$$

例如智能体 $a_{2}$ 下子 $(9,9)$ 对五子、活四、 单四、单三等准则没有贡献, 因为各要素要两两 比较, 其值不能为 0 , 故可设置其贡献为 1 。但 对孤子和防二子、防孤子有贡献, 可分别设置为 $99,100,99$ 。当 $j=9$ 时, 对应准则孤子, $a_{1}, a_{2}$, $a_{3}$ 对应的落子方案两两比较可得表 4 。 
表 4. 方案层 $P$ 的各要素对应准则层要素 $D_{9}$ 判断矩阵

\begin{tabular}{llll}
\hline$D_{9}$ & $P_{1}$ & $P_{2}$ & $P_{3}$ \\
\hline$P_{1}$ & 1 & $100 / 99$ & $100 / 99$ \\
$P_{2}$ & $99 / 100$ & 1 & 1 \\
$P_{3}$ & $99 / 100$ & 1 & 1 \\
\hline
\end{tabular}

对应矩阵的最大特征根为 $\lambda=3$, 对应的特征 向量归一化结果为

$$
\rho=\left(c_{1 \times 9}, c_{2 \times 9}, c_{3 \times 9}\right)^{T}=\left[\begin{array}{l}
0.3356 \\
0.3322 \\
0.3322
\end{array}\right]
$$

同理可算出其它的 $\boldsymbol{c}_{i \times j}$, 其中 $i=1,2,3$; $j=1,2, \cdots, 18$ 。

最后进行层次总排序。总排序计算结果为 $v_{1}=a_{1} \times b_{1} \times c_{1 \times 1}+a_{2} \times b_{2} \times c_{1 \times 2}+\cdots+a_{18} \times b_{18} \times c_{1 \times 18}=0.3334$

$v_{2}=a_{2} \times b_{2} \times c_{2 \times 1}+a_{2} \times b_{2} \times c_{2 \times 2}+\cdots+a_{18} \times b_{18} \times c_{2 \times 18}=0.3333$

$v_{3}=a_{1} \times b_{1} \times c_{3 \times 1}+a_{2} \times b_{2} \times c_{3 \times 2}+\cdots+a_{18} \times b_{18} \times c_{3 \times 18}=0.3333$

$a_{1}, a_{2}, a_{3}$ 落子方案排序为 $\left[v_{1}, v_{2}, v_{3}\right], \quad v_{1}$ 对应的 $a_{1}$ 执行方案为最优方案。

\subsection{2 时间中值法}

时间中值法是指选择落子不快不慢、时间在 中间的智能体的落子为选项。例如, 某一步智能 体 $a_{1}, a_{2}, a_{3}$ 依次落子坐标为 (9,9) $(9,8)(9,7)$, 用时分别为 $t_{1}, t_{2}, t_{3}, t_{1}<t_{2}<t_{3}$, 则取反应时间居 中的 $a_{2}$ 的落子坐标 $(9,8)$ 。

不失一般性, 设有 $m$ 个智能体参与对弯, 用 时由短到长排序为 $t_{1}, t_{2}, \cdots, t_{m}$ 。当 $m$ 为奇数 时, 落子时间居中的智能体的落子坐标下标是 $i=$ $(m+1) / 2$; 当 $m$ 为偶数时, 如果 $\mid t_{m / 2+1^{-}}\left(t_{1+} t_{m}\right)$ $|2|>\left(t_{1+} t_{m}\right) / 2-t_{m / 2} \mid$, 取 $i=m / 2$; 如果 $\mid t_{m / 2+1^{-}}\left(t_{1+} t_{m}\right)$ $|2|<\left(t_{1+}+t_{m}\right) / 2-t_{m / 2} \mid$, 取 $i=m / 2+1$ 。依此规则, 时 间中值法有横坐标 $X$ 和纵坐标 $Y$ 由式 (9) 确定。

$$
X=\boldsymbol{x}_{\boldsymbol{i}}, \quad Y=\boldsymbol{y}_{\boldsymbol{i}}
$$

\subsection{3 综合评价法}

综合评价法是指运用多个指标对多个参评 单位进行评价的方法。把每个智能体的落子位置 作为一个参评单位, 为了获取最后的胜利, 打败 电脑, 对每个落子位置进行评价, 选取得分较高 者作为这一步的落子位置。
评价指标体系的确定。根据智能体 $a_{1}, a_{2}$, $a_{3}$ 的落子位置 $p_{1}, p_{2}, p_{3}$, 对五子棋进攻和防守 的贡献, 把其分解为成五、活四、冲四、活三、 眠三、成二子、孤子、防成五、防活四、防冲四、 防活三、防眠三、防二子这 13 个要素, 其得分 分别用 $E_{1}, E_{2}, \cdots, E_{13}$ 表示, 其具体的含义在 上文的层次分析法中已经提到。

指标数据 $E_{1}, E_{2}, \cdots, E_{13}$ 的计算。根据各 个智能体的落子位置对已有的棋盘格局造成的 影响, 得到各自的指标数据。例如, 已有的棋盘 格局如图 4 中实心黑白球所示, 计算机执白子, 智能体执黑子。智能体 $a_{1}, a_{2}, a_{3}$ 分别落子为 $(9,7),(7,9),(7,6)$, 在图 4 中用空心圆来表示。 对于各个要素来说, 落子后如果不满足, 则赋值 为 0 , 例如 $a_{1}$ 落子后, 没有五子出现, 没有活四, 其得分为 0 , 即 $E_{1}=0, E_{2}=0$; 如果有一个活三出 现, 则赋值 100 , 如果有两个活三, 则赋值 200 , 以此类推, 其它各要素同理, 如 $a_{2}$ 落子后, 可以 成两个二子, 则 $E_{6}=200$, 可以防活三, 则 $E_{11}=100$ 。

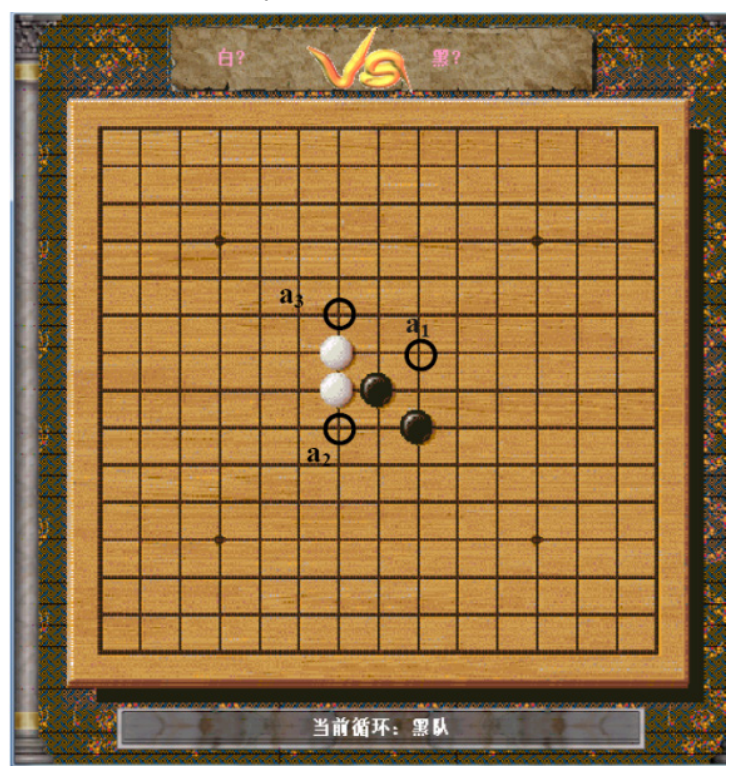

图 4. 现有的棋盘格局

确定评价指标权重 $\mu_{1}, \mu_{2}, \cdots, \mu_{13}$ 。 对于 成五、活四、冲四、活三、眠三、成二子、孤子、 防成五、防活四、防冲四、防活三、防眠三、防 二子这些要素来说, 成五子和防五子是最为关键 的, 活四、防活四居次, 而冲四、活三、防冲四、 防活三比眠三、成二子、孤子、防眠三、防二子 要重要些。其中进攻比防守要重要些。因为这些 相对重要性是确定的, 而且很明显, 可以据此尝 试直接确定权重值 $\mu_{1}, \mu_{2}, \cdots, \mu_{13}$ 。此次实验 中权重值为 


$$
\begin{aligned}
\mu & =\left(\mu_{1}, \mu_{2}, \cdots, \mu_{13}\right) \\
& =(100,50,20,20,10,10,5,90,45,15,15,8,8)
\end{aligned}
$$

于是, 在图 4 的棋盘格局中, 对落子 $(9,7)$ 的 综合评价值是

$$
v_{1}=\frac{E_{1} \times \mu_{1}+E_{2} \times \mu_{2}+\cdots+E_{13} \times \mu_{13}}{\mu_{1}+\mu_{2}+\cdots+\mu_{13}}=7.0175
$$

同理可计算得, 落子 $(7,9)$ 和 $(7,6)$ 的综合评价 值分别是 $v_{2}=8.7719, v_{3}=3.7594$ 。由于 $v_{2}>v_{1}>v_{3}$, 取 $v_{2}$ 对应的智能体 $a_{2}$ 的落子位置 $(7,9)$ 为黑方的 最终落子位置。

\section{3 实验步骤}

将开发测试完成的程序编译成可执行文件, 拷贝到 3 台计算机, 将它们连入互联网, 形成智 联网。联网后的每台计算机上, 均有五子棋棋盘, 显示智联网与计算机对弯中的棋盘格局。

选手根据自身对棋局的判断, 自由落子。参 与选手的落子均完成后, 嵌入智联网的数学模型 整合这些落子建议, 生成一个落子, 与计算机对 弯。

对弯开始前, 智能体 (选手) $a_{1}, a_{2}, a_{3}$ 输 入昵称注册进入游戏, 以层次分析法作为信息处 理模型，对弯一局，接着以时间中值法对弯一局， 再以综合评判法对栾一局; 接着, 再以层次分析 法对弯, 如此循环, 三种信息处理模型各进行 5 局。

由于参与实验的智能体在不同时间段内因 为疲倦、外界干扰等原因对实验的投入程度不 同, 各个模型循环进行可以降低这些外在因素对 实验结果的影响。

\section{4 结果与分析}

根据上述实验步骤, 选用层次分析法、时间 中值法和综合评价法作为信息处理模型时的五 子棋对恋结果如表 5 所示。

从是否闯关成功来看, 综合评价法 3 赢 2 输, 层次分析法和时间中值法都是 2 赢 3 输, 综合评 价法这个模型在处理 3 个智能体的落子信息时效 果要好些。从总步数来看, 综合评价法最少步数 20 , 最大 49 , 平均步数 30.6 , 赢局平均步数 38 , 输局平均步数 20 , 在 3 种方法中所用步数最少, 且最为集中稳定。层次分析法最少步数 23 , 最大 步数 94 , 平均每局步数 52 , 赢局平均步数 47 , 输局平均步数 55 , 则起伏最大, 平均用步数最多。 时间中值法最少步数 18 , 最多步数 59 , 平均步
数为 35.6 , 赢局平均步数 52 , 输局平均步数为 25 。时间上, 层次分析法平均用时 866355.6 毫秒, 综合评价法 427960 毫秒，时间中值法 495335.6 毫秒，和其所用步数呈正相关。

对比三种信息处理模型, 发现层次分析法和 时间中值法在赢局中所用步数相差不大, 但都比 综合评价法要多, 赢得较为艰难; 在输局中, 中 值法能坚持的步数很少, 而层次分析法的步数较 多, 说明后者抗输能力较强。

表 5 . 实验结果

\begin{tabular}{ccccc}
\hline $\begin{array}{c}\text { 整合模 } \\
\text { 型 }\end{array}$ & 局 & $\begin{array}{c}\text { 黑子 } \\
\text { 输赢 }\end{array}$ & $\begin{array}{c}\text { 黑子总 } \\
\text { 步数 }\end{array}$ & $\begin{array}{c}\text { 本局总时间 (毫 } \\
\text { 秒) }\end{array}$ \\
\hline & 1 & 赢 & 71 & 1084193 \\
层次分 & 2 & 赢 & 23 & 153469 \\
析法 & 3 & 输 & 34 & 463109 \\
& 4 & 输 & 94 & 2020275 \\
& 5 & 输 & 38 & 610732 \\
\hline & 1 & 输 & 18 & 95174 \\
时间中 & 2 & 赢 & 45 & 616642 \\
值法 & 3 & 输 & 34 & 429502 \\
& 4 & 输 & 22 & 429033 \\
& 5 & 赢 & 59 & 906327 \\
\hline & 1 & 输 & 20 & 242696 \\
综合评 & 2 & 赢 & 29 & 328917 \\
判法 & 3 & 赢 & 49 & 655946 \\
& 4 & 赢 & 35 & 511325 \\
& 5 & 输 & 20 & 400916 \\
\hline
\end{tabular}

实验表明，在解决五子棋决策问题时综合评 价法最好，层次分析法其次，时间中值法居三。 但考虑到时间中值法的简单性，整体上层次分析 法与时间中值法相差无几。

\section{1. 结论和讨论}

自然灾害风险分析中存在着假设的统计规 律难以证实、分析结果的可靠性难以保证等问 题, “实验风险学”试图通过实验来检验相关的风 险评估方法, 为解决该问题探索一条路径。伴随 着网络技术的飞速发展, 建立在线风险实验平台 成为一种趋势。而“智联网”的提出, 为其提供了 重要的工具。

本文首次提出在智联网平台上对自然灾害 风险分析中常用的整合型模型进行测试，对模型 进行优劣比较, 以五子棋人机大赛为例, 把多个 智能体在线提供的避免失败、力图闯关的信息用 
层次分析法和综合评价法进行整合, 并以简单的 取中法作为实验的参照, 提出了一种检验风险评 估模型的在线智联网实验方法。根据实验结果, 综合评价法战绩最好, 层次分析法整体效果只比 时间中值法稍好些。

这是用计算机网络实验平台试图解决风险 分析结果可靠性问题的一次有益的探索, 利用网 络平台, 可以大大提高这类实验的效率, 降低成 本。

\section{致谢}

本研究得到了国家自然科学基金资助项目 （No. 41471424）的资助; 北京崇安智联科技服 务有限责任公司部分资助。

\section{参考文献}

[1] 韩广才,张耀良. 一类广义力学系统的能量方程. 哈尔滨工程大学学报, 2001, 22 (4): 69-71.

[2] Cox L A Jr. Confronting deep uncertainties in risk analysis. Risk Analysis, 2012, 32(10): 1607-1629.

[3] Borsuk M E, Tomassini L. Uncertainty, imprecision, and the precautionary principle in climate change assessment. Water Science and Technology. 2005, 52(6): 213-25.

[4] Cordier A1, Gohin J, Krebs S, Rault A. Dynamic impacts of a catastrophic production event: the foot-and-mouth disease case. Risk Analysis, 2013, 33(3): $480-492$.
[5] Mitkus R J, King D B, Walderhaug M O, Forshee R A. A comparative pharmacokinetic estimate of mercury in U.S. infants following yearly exposures to inactivated influenza vaccines containing thimerosal. Risk Analysis, 2014, 34(4): 735-750.

[6] Slob W, Bakker M I, Biesebeek J D, Bokkers B G.. Exploring the uncertainties in cancer risk assessment using the integrated probabilistic risk assessment (IPRA) approach. Risk Analysis, 2014, 34(8): 1401-22.

[7] März R. Fine decouplings of regular differential algebraic equations. Results in Mathematics, 2004, 46 (1-2): 57-72.

[8] Diethelm K. An algorithm for the numerical solution of differential equations of fractional order. Electronic Transactions on Numerical Analysis, 1997, 5(1): 1-6.

[9] 肖静宇. 几类分数阶微分方程的数值方法研究. 哈尔滨：哈尔滨工业大学, 2013.

[10] Beylkin G. On the representation of operators in bases of compactly supported wavelets. SIAM Journal on Numerical Analysis, 1992, 6(6): 1716-1740.

[11] Marinescu D C. Cloud Computing: Theory and Practice. San Francisco: Morgan Kaufmann Publishers Inc., 2013.

[12] Huang C F. Experimental riskology: a new discipline for risk analysis. Human and Ecological Risk Assessment, 2013, 19(2): 389-399.

[13] Huang C F. Internet of intelligences can be a platform for risk analysis and management. Human and Ecological Risk Assessment, 2015, 21(5): 1395-1409. 